\title{
Syndrome anticholinergique central en période postopératoire
}

\section{The central anticholinergic syndrome in the postoperative period}

\author{
J. RUPREHT, B. DWORACEK \\ Anaesthesia Pharmacology Research School of Medicine, Erasmus University, Rotterdam, Netherlands
}

\begin{abstract}
NDLR. - Il a paru intéressant à la rédaction des AFAR de publier, avec l'accord des intéressés, la traduction de ce texte qui constitue le chapitre 95 de la $5^{e}$ édition de "General Anaesthesia " éditée par JF NunN, JE UtTing et BR R BRown Jr. Le lecteur trouvera une analyse de ce livre dans ce numéro.

Le syndrome anticholinergique central survenant en phase de réveil postanesthésique est peu connu des médecins anesthésistes, français notamment. Il est vrai, que s'agissant essentiellement d'un diagnostic d'élimination, la fréquence réelle de cc syndrome est difficile à appréhender. La lecture de l'excellent travail des docteurs Rupreht et Dworacek contribuera à mieux faire connâtre ce syndrome qui répond favorablement à la physostigmine (ésérine). Actuellement, cet alcalö̈de est uniquement disponible en France à la Pharmacie Centrale des Hôpitaux de Paris.
\end{abstract}

\begin{abstract}
RÉSUMÉ: Le syndrome anticholinergique central (SAC) comporte des signes centraux (somnolence, confusion, amnésie, agitation, hallucination, dysarthrie, ataxie, délire, stupeur, coma) et des signes périphériques (sécheresse buccale et/ou cutanée, tachycardie, troubles visuels et miction difficile). Il survient quand les sites anticholinergiques centraux sont occupés par des agents spécifiques ou en cas de libération insuffisante d'acétylcholine. Le SAC peut être déclenché par le sulfate d'atropine, l'hyoscine (scopolamine), la prométhazine (phénergan), les benzodiazépines, les opiacés, l'halothane, l'enflurane, la kétamine. La fréquence du SAC postopératoire dépend du type et de la dose d'anesthésiques, de l'acte pratiqué, de l'état du patient et des critères diagnostiques. Sa fréquence est proche de $10 \%$ après une anesthésie générale, de $4 \%$ après une anesthésie régionale avec sédation associée. Le diagnostic différentiel du SAC comporte notamment un surdosage en agents anesthésiques ou une modification de leur pharmacocinétique, des troubles de l'équilibre hydro-électrolytique, acide-base, une hypoglycémie, une hypoxie, une hypercapnie, une hypocapnie, une hyperthermie, une hypothermie, des désordres hormonaux, des lésions neurologiques en relation avec l'intervention, une embolie, une hémorragie, un traumatisme. Le diagnostic de SAC est souvent un diagnostic d'exclusion et n'est en fait porté qu'après obtention d'une réponse thérapeutique positive à la physostigmine, anticholinestérasique d'action centrale.
\end{abstract}

Dans les suites immédiates d'une intervention sous anesthésie générale, le rétablissement complet de l'ensemble des fonctions vitales peut être retardé ou perturbé. Actuellement l'anesthésie est généralement conduite de manière à permettre au patient de retrouver aussi rapidement que possible l'ensemble de ses facultés. Néanmoins, parmi les agents anesthésiques couramment utilisés, certains peuvent perturber la phase de réveil par suite de leurs effets anticholinergiques sur le système nerveux central. Ces effets qui constituent le syndrome anticholinergique central (SAC), se différencient des autres troubles de la phase de réveil et peuvent être corrigés par un anticholinestérasique d'action centrale, car franchissant la barrière hémato-encéphalique, telle que la physostigmine (ésérine).

\section{1. ÉTIOLOGIE ET MÉCANISMES DU SYNDROME ANTICHOLINERGIQUE CENTRAL}

Dans tous les territoires du cerveau, existent des synapses cholinergiques facilitatrices et inhibitrices. A leur niveau, la transmission peut être bloquée par des anticholinergiques à action centrale ou diminuée par les agents qui interfèrent avec la libération ou le turnover de l'acétylcholine (ACh). Des molécules dépourvues d'effets anticholinergiques primaires peuvent aussi réduire indirectement l'activité cholinergique centrale en modulant l'action d'autres transmetteurs tel l'acide gammaamino butyrique (GABA) intervenant dans l'activité cholinergique. De ce fait, on peut observer une grande variété de symptômes et de signes d'activité anticholinergique centrale. 


\subsection{Vigilance, état d'éveil et comportement}

Il est prouvé qu'à l'étage cortical, le niveau de conscience est étroitement dépendant de l'activité neuronale spontanée et répétitive, elle-même renforcée par l'ACh. Ces processus dépendent de l'activité d'un système cholinergique ascendant dénommé système réticulaire d'éveil [21]. Les réponses d'éveil à divers stimuli sont inhibées par des anticholinergiques telle l'atropine, ou renforcées par des substances cholinergiques d'action centrale, telle la physostigmine [36]. Le réveil comportemental est un processus complexe qui semble dépendre de facteurs autres que la seule ACh [20]. Le réveil cortical électroencéphalographique et le réveil comportemental peuvent être dissociés par l'atropine, montrant ainsi que les anticholinergiques modifient les fonctions cérébrales servant de support à la mémoire, l'apprentissage et la perception [25]. Cette soi-disant dissociation comportement-électroencéphalogramme [52] peut probablement s'expliquer par le fait que le message sensoriel est transmis à travers différentes voies avant d'atteindre le site d'intégration corticale. Une partie des synapses de transmission sont de type cholinergique donc susceptibles d'être bloquées par les anticholinergiques. De ce fait, seule une information partielle atteint le cortex cérébral. Ce blocage anticholinergique partiel de l'information afférente est responsable d'une réponse perturbée aux stimuli externes. A titre d'exemple ceci se traduit en phase de réveil par un état d'agitation en cas de douleur ou par un laryngospasme en présence de sécrétions. En revanche, chez un patient conscient, la douleur ne s'accompagne pas d'agitation et la présence de sécrétions entraîne plutôt soit une toux soit un réflexe de déglutition.

\subsection{Mémoire}

Les anticholinergiques d'action centrale peuvent interférer avec la mémoire et entraîner habituellement une amnésie ou des difficultés de concentration. Des travaux expérimentaux ont montré que la transmission cholinergique centrale est essentielle pour le processus de la mémoire $[25,37,51]$. Les déficits de mémoire peuvent avoir pour cause un blocage des synapses muscariniques centrales par les anticholinergiques ou une diminution d'activité de la choline acétyltransférase avec une diminution correspondante de l'ACh dans l'hippocampe [27]. De plus, certains anesthésiques peuvent aussi diminuer la libération d'ACh dans le cerveau [19]. L'effet thérapeutique bénéfique de la physostigmine pour stimuler la mémoire des sujets amnésiques, est une autre preuve du rôle de la transmission cholinergique centrale dans le processus de la mémoire [31].

\subsection{Effets circulatoires et respiratoires}

Après administration de physostigmine, le tonus cardiovasculaire est généralement augmenté [41]. Ceci peut s'expliquer par des résultats expérimentaux montrant que cet alcaloïde est responsable d'une stimulation hémodynamique d'origine centrale, transmise en périphérie par le système nerveux sympathique [47]. La physostigmine entraîne une réponse vasopressive directe, centrale, $\mathrm{ACh}-$ dépendante [4]. Il semble que l'activité cholinergique centrale soit essentielle pour la réaction au stress et intervienne dans la physiopathologie de l'hypertension artérielle [18].

Le rôle de la transmission cholinergique centrale dans le contrôle de la respiration a été démontré suite à l'administration par voie générale ou intracisternale d'inhibiteurs centraux de la cholinestérase qui permettent d'antagoniser la dépression respiratoire morphinique [29]. Il semble que les effets dépresseurs respiratoires et cardiovasculaires de la morphine, mais non l'analgésie, résultent d'une inhibition de la libération d'ACh par les neurones du système nerveux central [48]. La physostigmine semble pouvoir réverser l'effet dépresseur respiratoire des opiacés et rétablir la sensibilité du centre respiratoire au gaz carbonique, probablement en élevant la concentration d'ACh dans le cerveau, réduite par les opiacés [43]. La séparation entre analgésie morphinique et dépression respiratoire se base sur la mise en jeu du mécanisme endophinergique $\mu-2,[23]$. Quel que soit le mécanisme incriminé il est cliniquement important qu'une augmentation de l'ACh dans le cerveau antagonise les effets anticholinergiques centraux ainsi que la dépression respiratoire morphinique. Alors que les fonctions mentales de l'opéré se rétablissent, l'antinociception postopératoire n'est pas diminuée mais plutôt augmentée $[41,50]$.

\subsection{Antinociception}

Des mécanismes cholinergiques muscariniques centraux interviennent dans le fonctionnement de la voie de la douleur chez le rat [22]. Chez les volontaires sains la physostigmine $(1 \mathrm{mg})$ augmente de $30 \%$ le seuil de douleur à la chaleur radiante [11]. L'oxotremorine et la physostigmine augmentent de façon dose-dépendante le temps de réaction au test de la plaque chauffante chez la souris [6]. Bien que l'on admette généralement la participation de mécanismes cholinergiques centraux dans le processus d'antinociception, ceux-ci ne semblent pas intervenir dans le cas de la physostigmine. Il est établi que l'action analgésique de la physostigmine dépend de la transmission centrale faisant intervenir la sérotonine (5 hydroxytryptamine) et non de son action cholinergique centrale $[1,33]$. 


\subsection{Etat d'anesthésie}

L'ACh a été incriminée dans la genèse de l'état d'anesthésie [13]. Les études sur la concentration et le turnover d'ACh dans le cerveau du rat en cours d'anesthésie par l'halothane, l'enflurane ou la kétamine montrent l'absence de modification de cette concentration. D'un autre côté, seul l'halothane diminue le turnover de l'ACh dans tous les territoires du cerveau, alors que l'enflurane et la kétamine ne le font que dans quelques rares sites cérébraux [28]. Des opiacés tels que la morphine et la péthidine diminuent la libération d'ACh dans le cortex cérébral du chat, alors que la naloxóne la restaure [19]. Néanmoins l'analgésique morphinique ne dépend pas de la libération d'ACh car ce type d'analgésie ne peut être bloqué par l'administration d'anticholinergique [30]. Le rôle joué par le blocage de la transmission cholinergique centrale dans la genèse de l'état d'anesthésie est remis en question. En effet, en cours d'anesthésie, l'injection continue de physostigmine ne modifie ni l'induction ni l'entretien de celle-ci [9]. De plus, l'usage préférentiel d'anticholinergiques à action périphérique n'a apparemment pas modifié les posologies habituellement utilisées en anesthésie. Enfin, chez le chien, la physostigmine diminue de façon dose-dépendante la concentration alvéolaire minimale (MÁC) lors d'une anesthésie à l'halo-

Tableau 1. - Manifestations du syndrome anticholinergique central chez l'homme

\begin{tabular}{|l|l|}
\hline Central & \\
Agitation & Instabilité émotionnelle \\
Amnésie & Incoordination motrice \\
Ataxie & Nausées, vomissements occasionnels \\
Asynergie & Hyperpyrexie (origine centrale) \\
Sensibilité émoussée & Hyperalgésie \\
Confusion & Diminution du temps de réaction \\
Excitation & Dissociation EEG-comportement \\
Somnolence & Convulsions \\
Coma & Diminution pouvoir de concentration \\
Appréhension & Stimulation/dépression ventilatoire \\
Hallucinations & Fatigue, épuisement \\
Illusions & Mouvements stéréotypés \\
Delirium & Troubles mentaux de moyenne ou longue \\
& durée \\
Manifestations paranoìdes & \\
Périphérique & \\
Bouche sèche & \\
Peau sèche & \\
Soif & Vision rapprochée trouble \\
Ralentissement cardiaque & Mydriase, cycloplégie \\
(atropine à faible dose) & Photophobie \\
Tachycardie, palpitations & Troubles de la parole \\
(atropine à forte dose) & Difficulté de miction \\
Troubles du rythme cardiaque & Diminution de la motilité gastro-intestinale \\
\hline & Rougeurs cutanées \\
& Hyperpyrexie (origine périphérique) \\
\hline
\end{tabular}

thane [15]. Dans des conditions similaires, la physostigmine donne naissance à des signes de réveil électroencéphalographique [38]. Néanmoins, il n'existe à l'heure actuelle aucune preuve que la physostigmine augmente le niveau de vigilance en cours d'anesthésie chez l'homme. Ceci indique que les effets anticholinergiques centraux, survenant en période postopératoire, peuvent être considérés comme des effets secondaires indésirables, induits par les agents utilisés et justiciables d'un traitement approprié (tableau I).

\section{SYNDROME ANTICHOLINERGIQUE CENTRAL}

\subsection{Terminologie}

Les symptômes comportementaux et somatiques induits par les anticholinergiques d'action centrale sont nombreux et imprévisibles. Ils ont été regroupés, dans le cas de l'homme et de l'animal, sous le terme de SAC par Longo en 1966, dans sa revue sur les effets comportementaux et électroencéphalographiques de l'atropine et composés voisins [25]. Divers termes devenus obsolètes, ont été utilisés pour la description de certains aspects du $\mathrm{SAC}$, en particulier délire postanesthésique, dépression postanesthésique, état d'émergence, délire d'émergence, somnolence postopératoire, effets postanesthésiques indésirables. De tels termes sont purement descriptifs car ils se réfèrent à des troubles postopératoires d'origine très diverse et ne spécifient pas de la cause du trouble, lié à l'effet anticholinergique central des agents utilisés.

\subsection{Manifestations du syndrome anticholinergique central}

Le tableau clinique du SAC comporte essentiellement des manifestations d'origine centrale c'està-dire comportementales telles que somnolence, confusion, amnésie, agitation, hallucinations, dysarthrie, ataxie, délire, stupeur, coma. Ces signes peuvent être particulièrement accentués en cas d'intoxication par les anticholinergiques. Les fonctions cognitives, en particulier la prise de conscience des événements, la place et le passage du temps, sont généralement diminuées voire absentes. Souvent les opérés semblent conscients mais restent troublés et demandent continuellement où ils en sont. Les sujets agités et non coopérants, qui souffrent en fait d'un trouble de la mémorisation des événements proches, entrent aussi dans cette catégorie.

Les signes anticholinergiques périphériques accompagnant le SAC sont une sécheresse de la bouche et de la peau, des modifications du rythme cardiaque, des troubles visuels et des difficultés de 
miction. Les signes centraux et périphériques de l'action anticholinergique sont rassemblés dans le tableau I.

Il importe de noter que les anticholinergiques ont un index thérapeutique, c'est-à-dire un rapport dose efficace sur dose létale, élevé. En d'autres termes la plupart de leurs effets sont dose-dépendants pour leur survenue et leur intensité. De plus, à dose identique, un anticholinergique peut produire des symptômes d'intensité variable en fonction de la sensibilité individuelle vis-à-vis d'un agent donné.

Les manifestations du SAC peuvent être grossièrement regroupées en deux tableaux cliniques, l'un où prédomine un état d'hyperactivité, l'autre caractérisé par un état de dépression. Une telle subdivision facilite la reconnaissance d'un SAC. Néanmoins les effets anticholinergiques centraux réalisent une symptomatologie complexe, difficile à évaluer et à classer, en particulier au sortir de la salle d'opération. Il n'existe pas de règle générale permettant de prédire les effets postopératoires des drogues. On admet généralement que l'hyoscine (scopolamine) tend à déprimer les fonctions. Néanmoins une agitation motrice incontrôlée, particulièrement chez le sujet âgé, peut aussi être l'indice d'une dépression centrale partielle. En présence d'une douleur, d'une angoisse préalable, l'effet résiduel d'autres agents, ainsi que certaines affections associées peuvent modifier le tableau clinique induit par une perturbation de la transmission cholinergique centrale. La prise thérapeutique ou abusive de psychotropes est souvent un facteur favorisant la survenue d'un SAC en période postopératoire.

L'hyperpyrexie centrale est un symptôme du SAC [41]. Cette complication rare mais dangereuse peut ne pas être rattachée à sa cause chez un opéré déjà fébrile. Le centre de thermorégulation situé dans la partie rostrale de l'hypothalamus comporte de nombreuses synapses cholinergiques susceptibles d'être bloquées par les anticholinergiques et de ce fait, de perturber les mécanismes de thermolyse [8].

Les manifestations du SAC peuvent durer des heures, voire des jours. Elles peuvent parfaitement donner lieu à un tableau de troubles mentaux pendant la première semaine postopératoire [35]. Cependant jusqu'à présent, des troubles mentaux de durée moyenne ou prolongée, après une anesthésie et leur relation avec le SAC n'ont pas bénéficié d'une étude scientifique approfondie.

\subsection{Agents déclenchant un syndrome anticholinergique central}

De nombreux agents ont une activité anticholinergique centrale. Le SAC apparaît quand les sites cholinergiques centraux sont occupés par des agents spécifiques et quand la libération d'ACh est insuffisante. Sur le marché américain il existe au moins 500 agents capables d'avoir des effets anticholinergiques centraux [12]. Un SAC peut être déclenché par de nombreux alcaloïdes en particulier ceux de la belladone, les antidépresseurs, les antihistaminiques, les antipsychotiques, les antiparkinsoniens, les benzodiazépines, de nombreux agents anesthésiques, et la plupart des «drogues » psychotropes d'usage illicite (tableau II).

Tableau II. - Agents responsables d'un syndrome anticholinergique central
Antidépresseurs

Antihistaminiques

Antipsychotiques

Antispasmodiques

Antiparkinsoniens

Agents anesthésiques (Anesthésiques par inhalation compris)

Alcaloïdes de la belladone

Opiacés

Armes chimiques psychotropes

Tranquillisants, Benzodiazépines

Hallucinogènes (LSD, kétamine, mescaline, psilocybine)

Diverses armes chimiques sont capables de bloquer la transmission cholinergique centrale et d'entraîner une symptomatologie de SAC [25]. Comme les victimes de ces agents sont temporairement incapables d'agir, ces agents sont aussi appelés des incapacitants.

Tous les anticholinergiques ayant une structure d'amine tertiaire sont liposolubles et traversent rapidement la barrière hématoencéphalique. Tel est le cas des sulfates d'atropine et d'hyoscine (scopolamine). Leurs analogues méthylés, par suite de leur structure d'amine quaternaire, ne sont pas liposolubles et de ce fait sont dénués d'effets centraux. En pratique anesthésique courante, seuls les effets anticholinergiques périphériques sont réellement utiles. C'est pourquoi il faut préférer l'atropine ou l'hyoscine quaternaires à leurs analogues tertiaires.

Il existe peu d'arguments en faveur de l'idée généralement admise selon laquelle le sulfate d'atropine est plus sûr que l'hyoscine chez le sujet âgé. Leurs effets centraux sont sensiblement identiques, en particulier à posologie élevée [16], chez les patients déshydratés ou fébriles. Néanmoins, aux faibles posologies utilisées en anesthésie, l'hyoscine tertiaire semble moins déprimer les fonctions vitales que l'atropine, en particulier en ce qui concerne la perception. Ces impressions cliniques n'ont pas encore été confirmées par une étude détaillée des modifications mertales induites par ces agents.

Le SAC en relation avec une libération insuffisante d'ACh peut être observé après administra- 
tion de morphine ou de péthidine [19], d'halothane, d'enflurane ou de kétamine [28]. Le phénobarbital (Gardénal) à concentrations anesthésiques diminue la libération spontanée et provoquée d'ACh [24]. Ces effets sur le métabolisme de l'ACh peuvent se produire dans la totalité du cerveau ou rester limités à quelques territoires. Le mécanisme par lequel les anesthésiques gazeux ou intraveineux produisent les symptômes d'un bloc cholinergique central ne sont pas encore complètement expliqués. Néanmoins les effets favorables $\mathrm{du}$ traitement par la physostigmine pour corriger cette symptomatologie indiquent que l'anesthésie générale est capable d'inhiber la transmission cholinergique.

\subsection{Diagnostic différentiel du syndrome anticholinergique central}

Les signes du SAC sont proches (voire même identiques) de ceux d'autres états perturbant le réveil postanesthésique. Ainsi, un coma postanesthésique, un réveil retardé et agité peuvent être témoins d'un surdosage en anesthésiques ou d'une perturbation de leur pharmacocinétique, telle une réduction de leur catabolisme et de leur élimination. De plus, un tableau voisin du SAC peut être d'origine métabolique, en particulier une modification de la concentration sanguine d'électrolytes et de glucose. Un trouble de l'équilibre acide-base peut aussi influencer la perception. Au niveau cérébral, l'hypoxie, l'hypercapnie, l'hypocapnie, l'hyperthermie et l'hypothermie ainsi que les désordres hormonaux et la déshydratation peuvent modifier le comportement. Il peut exister des lésions nerveuses en rapport avec l'acte chirurgical, une embolie, une hémorragie ou un traumatisme. Néanmoins, comme la plupart des agents anesthésiques interfèrent probablement avec la transmission cholinergique centrale, il faut envisager de façon systématique qu'un réveil postanesthésique perturbé peut être partiellement ou totalement dû à un SAC. Le diagnostic est souvent un diagnostic d'exclusion. Il n'est réellement porté qu'après obtention d'une réaction positive à l'administration d'un anticholinestérasique à effet central.

A l'heure actuelle le diagnostic de SAC ne peut être obtenu par les examens complémentaires. Le recours à l'EEG a été préconisé mais cette méthode ne donne pas de résultats fiables par suite de la faible corrélation entre modification EEG et comportement clinique [5, 32].

\subsection{Fréquence du syndrome anticholinergique}

La survenue d'un SAC en période postopératoire dépend de nombreux facteurs, en particulier du type et de la dose d'agent anesthésique, du type de chirurgie, de l'état du patient et des critères d'inclusion pour affirmer un trouble de la fonction cholinergique centrale. Dans l'expérience des auteurs de ce travail, un SAC a été observé chez $9,4 \%$ des patients après anesthésie générale et chez 3,3\% après anesthésie loco-régionale associée à une sédation [41]. Un groupe a rapporté la présence d'un état d'excitation postanesthésique chez $6 \%$ des hommes et $5 \%$ des femmes, mais en excluant les états de dépression et de retard de réveil des critères d'inclusion dans le diagnostic de SAC [10]. La fréquence de survenue du SAC varie notablement d'un travail à l'autre en fonction des critères de diagnostic retenus.

Dans l'expérience des auteurs, la fréquence de survenue du SAC postopératoire se distribue harmonieusement quel que soit le sexe ou l'âge. Les sujets âgés tendent néanmoins à présenter plus souvent des signes de dépression, contrairement aux jeunes qui sont plus souvent agités. Ces différences dans les aspects cliniques revêtus par le SAC ont probablement contribué à la grande variation des fréquences de survenue rapportées par différents auteurs.

\section{TRAITEMENT DU SYNDROME ANTICHOLINERGIQUE CENTRAL}

Le traitement du SAC requiert l'augmentation de la concentration cérébrale d'ACh. Ceci peut être obtenu à l'aide de salicylate de physostigmine, de bromhydrate de galantamine [3], de 4-aminopyridine [39] ou de tacrine [26]. Ces agents peuvent pénétrer dans le cerveau et permettre la réaugmentation de la concentration d'ACh. La galantamine et la tacrine sont difficiles à obtenir et on dispose de peu d'information sur leur effet en cas de SAC. La 4-aminopyridine a une action très peu spécifique et de ce fait est peu adaptée au traitement [45].

\subsection{Salicylate de physostigmine: posologie et pharmacocinétique}

La physostigmine, inhibiteur tertiaire de la cholinestérase, peut être administrée par voie intraveineuse ou intramusculaire, la dose initiale optimale étant de $0,04 \mathrm{mg} \cdot \mathrm{kg}^{-1}$. En cas d'administration par voie intraveineuse, l'injection doit avoir lieu lentement de façon à obtenir une distribution harmonieuse dans la circulation. La vitesse d'injection ne doit pas dépasser le tiers de la dose totale initiale par minute pour prévenir la survenue de nausées et de salivation. L'expérience montre que la dose de physostigmine requise ne doit pas se baser sur l'âge mais sur le poids et l'intensité du SAC.

La physostigmine est rapidement hydrolysée, $1 \mathrm{mg}$ étant détruit chez l'adulte en deux heures [44]. Après injection intraveineuse, le début d'action est légère- 
ment plus rapide qu'après administration intramusculaire. Par ailleurs, la physostigmine est rapidement éliminée du compartiment plasmatique, les demi-vies de distribution et d'élimination plasmatique étant respectivement de 2,3 et de 22 minutes [14]. Cette courte demi-vie de distribution confirme l'expérience antérieure selon laquelle l'injection prudente et lente diminue notablement la fréquence de survenue des effets secondaires intestinaux ou cardiaques. Il a aussi été montré qu'une concentration plasmatique de $3-5 \mathrm{ng} \cdot \mathrm{ml}^{-1}$ doit être dépassée pour obtenir une réversion clinique adéquate d'un SAC postopératoire. Ceci correspond à au moins $2 \mathrm{mg}$ de physostigmine chez l'adulte et aux doses recommandées dans les travaux antérieurs [41]. Chez les patients intoxiqués par un anticholinergique, la dose de physostigmine requise peut dépasser $0,04 \mathrm{mg} \cdot \mathrm{kg}^{-1}$, en fonction des signes d'amélioration en cours de traitement. Les antidépresseurs tricycliques tendent à perturber la conduction intracardiaque qui de ce fait doit être soigneusement monitorée pendant le traitement par la physostigmine [7].

\subsection{Réversion des manifestations du syndrome anticholinergique central}

Le début d'action de la physostigmine est rapide (0,5-3 minutes) quand le SAC est dû à l'atropine, l'hyoscine ou la prométhazine. Après administration de physostigmine, un patient déprimé ou délirant devient alerte et coopérant. Le début d'action peut être plus tardif (5-15 minutes) si le SAC résulte de l'effet combiné d'anticholinergiques et d'autres dépresseurs centraux. Dans certains cas la physostigmine réverse seulement les signes en relation avec le SAC mais non ceux en relation avec l'effet résiduel d'autres agents. En pareil cas, l'agitation disparaît mais la dépression liée à l'effet résiduel des anesthésiques persiste. En d'autres termes, le réveil n'est pas raccourci mais l'état du patient est bien meilleur. Ce résultat correspond à celui obtenu chez l'animal d'expérience, à savoir que la physostigmine n'antagonise pas spécifiquement la somnolence due à l'halothane, sauf de façon indirecte par son effet d'activation adrénergique [15].

\subsection{Durée d'effet de la physostigmine}

La durée d'action moyenne d'une dose de $0,04 \mathrm{mg} \cdot \mathrm{kg}^{-1}$ de physostigmine est comprise entre 90 et 120 minutes. Chez les opérés qui en ont bénéficé, les signes de SAC ne réapparaissent habituellement pas. Dans le cas contraire, peut être effectuée une nouvelle injection correspondant à la moitié de la dose initiale. Les patients intoxiqués requièrent une surveillance prolongée et parfois des doses répétées de physostigmine selon le type de poison et la sévérité de l'intoxication.

La relative courte durée d'action de la physo- stigmine a un grand intérêt pour le diagnostic différentiel de troubles susceptibles d'être liés à des effets anticholinergiques centraux. Ainsi, dans une intoxication de cause inconnue, la présence d'anticholinergiques peut être exclue si l'administration de physostigmine n'entraîne pas une certaine amélioration de l'état du patient. Un essai thérapeutique avec la physostigmine est sûr sauf dans les conditions rappelées ci-dessous.

L'administration d'une dose-test de physostigmine peut aussi permettre de progresser dans le diagnostic différentiel d'un coma secondaire à une intervention neurochirurgicale [40]. Enfin, la durée d'action limitée de la physostigmine en fait aussi un moyen pharmacologique utile pour un test de réveil en cours d'intervention neurochirurgicale ou orthopédique [46].

\subsection{Effets secondaires de la physostigmine}

La physostigmine entraîne chez moins de $20 \%$ des opérés des nausées qui répondent rapidement à un anti-émétique. D'autres effets secondaires telles une tachycardie et une augmentation de la pression artérielle restent généralement modérés. Chez l'hypertendu la physostigmine peut déterminer une poussée hypertensive réclamant un traitement [17]. Il faut aussi se rappeler qu'un obstacle mécanique au niveau du tube digestif ou des voies urinaires, est une contre-indication à ce médicament. Habituellement la physostigmine n'interfère pas avec l'effet cholinergique périphérique des anticholinestérasiques donnés pour la réversion de la curarisation. En d'autres termes, l'administration de physostigmine après celle de néostigmine n'entraîne généralement pas de problème particulier. Il faut cependant se rappeler que la sécurité, en cas d'utilisation d'un anticholinestérasique, dépend en grande partie de la normalité des gaz du sang [34]. Enfin l'adjonction d'anticholinergiques n'est pas requise dès lors que la physostigmine est administrée lentement.

La physostigmine et la néostigmine sont tous deux des inhibiteurs spécifiques de la cholinestérase mais leurs effets pharmacologiques diffèrent notablement. Le fait de traverser la barrière hématoencéphalique est une caractéristique importante permettant à la physostigmine d'agir sur un certain nombre de fonctions centrales telles que le contrôle cardio-vasculaire, la mémoire, la vigilance et la nociception (tableau III). Les différences avec la néostigmine sont encore plus évidentes en présence d'autres agents ayant une activité centrale tels les anticholinergiques, les opiacés ou les antidépresseurs. De plus, à dose excessive la néostigmine ne détermine qu'une augmentation quantitative de son action périphérique, alors que la physostigmine est capable d'induire par ailleurs une séquence d'effets qualitativement différents par suite de son action centrale. 
Tableau III. - Physostigmine et néostigmine : propriétés comparatives

\begin{tabular}{lcc}
\hline & Physostigmine & Néostigmine \\
\hline Passage barrière hématoencéphalique & + & - \\
Efficacité sur le SAC & + & - \\
Effet sur : & légère augmentation & diminution \\
Fréquence cardiaque & augmentation & diminution \\
Pression artérielle & + & ++ \\
Motilité gastro-intestinale & faible antagonisme & ++ \\
Bloc neuro-musculaire & ++ & puissant antagonisme \\
Antinociception & + & + \\
Facilitation de la mémoire & antagonisme & - \\
Vigilance & antagonisme & - \\
Dépression respiratoire par opiacés & + & - \\
Somnolence par les opiacés & légère diminution & + \\
Nausées & & - \\
MAC & & + \\
\hline
\end{tabular}

La physostigmine ne doit pas être utilisée chez les patients ayant un traumatisme crâniocérébral fermé ou une intoxication barbiturique. Ces états se caractérisent en effet par une concentration d'ACh élevée dans le tissu cérébral et l'adjonction de physostigmine peut alors entraîner des convulsions. En cas de dystrophie myotonique et d'intoxication par les agents cholinergiques, la physostigmine est contre-indiquée.

\subsection{Autres effets de la physostigmine}

Quand le diagnostic de SAC est correctement posé, la physostigmine améliore l'état du patient et peut raccourcir la phase de réveil. En plus de la réversion des signes de SAC résultant d'un blocage des récepteurs muscariniques centraux ou d'une réduction de la quantité d'ACh libérée, la physostigmine a aussi un effet antagoniste sur la dépression respiratoire induite par les morphiniques.
Enfin, en plus de la réversion de la sédation postopératoire, la physostigmine peut aussi entraîner une diminution subjective et objective de la nociception [50]. Cet effet pourrait découler de l'interférence de la physostigmine avec le système de transmission sérotoninergique [1] et n'est réversé ni par l'atropine ni par la naloxone [49, 53]. Cet effet complémentaire de la physostigmine est bénéfique en période postopératoire immédiate.

Contrairement aux résultats d'un travail déjà ancien concluant qu'une dose de physostigmine atteignant $4 \mathrm{mg}$ n'avait pas d'effet notable sur le bloc neuromusculaire des curares [2], une étude plus récente a montré que cet agent était capable de réverser un tel bloc mais apparemment par un mécanisme différent de celui de la néostigmine [42]. Cependant les deux travaux déconseillent l'utilisation de la seule physostigmine pour réverser un bloc neuromusculaire. En principe, un bloc résiduel doit être traité avant d'évaluer les manifestations d'un SAC.

Tableau IV. - Propriétés comparatives de quelques anticholinergiques

\begin{tabular}{lcccc}
\hline & Atropine sulfate & Methylatropine & Glycopyrrolate & Hyoscine \\
\hline Structure & Tertiaire & Quaternaire & Quaternaire & Tertiaire \\
Liposolubilité & + & - & - & - \\
Déclenchement SAC & + & - & - & + \\
Effets sur & & - & + & très faible dose \\
Bradycardie & très faible dose & ++ & ++ \\
Tachycardie (dose dépendante) & + & + & + & + \\
Autres effets anticholinergiques périphériques & + & + & + \\
Usage préférentiel en anesthésie & - & & + \\
\hline
\end{tabular}




\section{PRÉVENTION DU SYNDROME ANTICHOLINERGIQUE CENTRAL POSTOPÉRATOIRE}

La survenue du SAC postopératoire peut être prévenue par le respect des éléments suivants : éviter les anticholinergiques d'action centrale tels le sulfate d'atropine, le bromhydrate d'hyoscine ou la prométhazine ; éviter les anesthésiques tendant à entraîner un SAC ; utiliser les anticholinergiques d'action périphérique tels la méthylatropine ou le glycopyrrolate (tableau IV) ; utiliser plus largement les anticholinestérasiques d'action centrale.

Dans l'expérience des auteurs de ce travail la fréquence de survenue du SAC rapportée antérieurement [41] a diminué entre temps de deuxtiers grâce à la suppression des anticholinergiques de la prémédication et la substitution du sulfate d'atropine par la méthylatropine dépourvue d'effet central. Une autre approche a consisté à utiliser le glycopyrrolate à la place des anticholinergiques classiques. Chez les sujets exposés au risque de SAC postopératoire (comme la plupart des patients utilisant des agents psychotropes), le développement d'un SAC peut être prévenu par l'administration de physostigmine avant la fin de l'anesthésie.

\section{BIBLIOGRAPHIE}

1. Aiello-Malmberg P, Bartolini A, Bartolini R, Galli A. Effects of morphine, physostigmine and raphe nuclei stimulation on 5-hydroxytryptamine release from the cerebral cortex of the cat. Br J Pharmacol, $65: 547-555,1979$.

2. Baraka A. Antagonism of neuromuscular block by physostigmine alone in man. $B r J$ Anaesth, 50 : 1075-1077, 1978.

3. Baraka A, Harik S. Reversal of central anticholinergic syndrome by galanthamine. J Am Med Ass, 238 : 22932294, 1977.

4. Brezenoff HE. Centrally induced pressor responses to intravenous and intraventricular physostigmine evoked via different pathways. Eur J Pharmac, 23 : 290-292, 1973.

5. Commin P, Bismuth C, Gaultier M, Mellerio F. Apport de la corrélation électroencéphalographie-clinique à l'activité cholinergique centrale de la physostigmine. Agressologie, $19: 287-292,1978$.

6. Cox B, ThA SJ. The antinociceptive activities of oxotremorine, physostigmine and dyflos. $J$ Pharm Pharmacol, 24 : 547-551, 1972

7. Daunderer M. Physostigmine salicylate as an antidote. Int $J$ Clin Pharmac Ther Tox, 18 : 523-535, 1980.

8. De MaAR EJW. Site and mode of action in the central nervous system of some drugs used in the treatment of Parkinsonism. Arch Int Pharmacodyn Ther, 105 : 349-365, 1956.

9. DworaceK B, Rupreht J. Is central cholinergic blockade essential for the anaesthetic state? Eur J Anaesthesiol, 1 : 154P-155P, 1984.

10. Eckenhoff JE, Kneale DH, Dripps RD. The incidence and etiology of postanesthetic excitement. Anesthesiology, 22: 667-673, 1961.

11. Flodmark $S$, Wramner $T$. The analgetic action of morphine, eserine and prostigmine studied by a modified
Hardy-Wolff-Goodell method. Acta Physiol Scand, 9 : 8896, 1945.

12. Granacher RP, Baldessarini RI. Physostigmine. Arch Gen Psychiatry, 32 : 375-380, 1970.

13. HANIN I. Anesthetics and central cholinergic function - a perspective. Anesthesiology, 48 : 1-3, 1978.

14. Hartvig P, Wiklund L, Lindsström B. Pharmacokinetics of physostigmine after intravenous, intramuscular and subcutaneous administration in surgical patients. Acta Anaesthesiol Scand, 38 : 177-182, 1986.

15. Horrigan RW. Physostigmine and anesthetic requirement for halothane in dogs. Anesth Analg, 57 : 180-185, 1978.

16. InNES IR, NiCKERSON M. Drugs inhibiting the action of acetylcholine on structures innervated by postganglionic parasympathetic nerves ( $\mathrm{pp}$ 521-525). In: The pharmacological basis of therapeutics. $3^{\text {rd }}$ edit, LS Goodman, A. Gilman eds. Macmillan, New York, 1965.

17. JaNOWSKY DS, Risch SC, HueY LY. Central cardiovascular effects of physostigmine in humans. Hypertension, $7: 140$ 145,1985 ,

18. JANOwSky DS, Risch SC, Huey L, Judd LE, Rausch J. Central physostigmine-induced cardiovascular and behavioral changes : toward an acetylcholine hypothesis of stress. Psychopharmac Bull, 19 : 675-681, 1983.

19. Jhamandas K, Phillis JW, Pinsky C. Effects of narcotic analgesics and antagonists on the in vivo release of acetylcholine from the cerebral cortex of the cat. Br J Pharmacol, 43 : 53-66, 1971.

20. Krnjevic K. Chemical transmission and cortical arousal. Anesthesiology, 28 : 100-105, 1967.

21. KrnjeviC K. Central cholinergic pathways. Fed Proc, 28 : 113-120, 1969.

22. LEwis JW, Cannon JT, LiEbeskind JC. Involvement of central muscarinic cholinergic mechanisms in opioid stress analgesia. Brain Res, $270: 289-293,1983$.

23. Ling GSF, Siegel K, Lockhart SH, Pasternak GW. Separation of opioid analgesia from respiratory depression : evidence for different receptor mechanisms. I Pharmacol Exp Ther, 232 : 149-155, 1985.

24. LitTle HJ, Paton WDM, SMITH EB. Effects of anesthetics and of helium pressure on acetylcholine release ( $\mathrm{pp}$ 457461). In : Molecular Mechanisms of Anesthesia (Progress in Anesthesiology, vol 2), Br Fink ed. Raven Press, New York, 1980.

25. LONGo VG. Behavioral and electroencephalographic effects of atropine and related compounds. Pharmacol Rev, 18 : 965-996, 1966.

26. Mendelson G. Central anticholinergic syndrome reversed by tetrahydroaminacrine (THA). Med J Aust, 2: 906, 1975.

27. Micheau J, Destrade J, Jaffard R. Physostigmine reverses memory deficits produced by pretraining electrical stimulation of the dorsal hippocampus in mice. Behav Brain Res, 15 : 75-81, 1985.

28. Ngai SH, Cheney DL, Finck AD. Acetylcholine concentrations and turnover in rat brain structures during anesthesia with halothane, enflurane and ketamine. Anesthesiology, 48: 4-10, 1978.

29. Paskov DS, Dobrev H, Nikiforov N. Antagonistic action of nivaline and morphine upon the respiratory center (pp 113-123). In : Proceedings of the Second International Pharmacological Meeting, Prague, vol 2, Pergamon Press, Oxford, 1963.

30. Pert A. The cholinergic system and nociception in the primate : interactions with morphine. Psychopharmacologia, 44 : 131-137, 1975.

31. Peters BH, Levin HS. Memory enhancement after physostigmine treatment in the amnestic syndrome. Arch Neurol, 34: 215-219, 1977. 
32. Pichlmayr I, Lips U. Atropin-Effekte im Elektroenzephalogramm. Anaesthesist, 29 : 249-253, 1980.

33. Pleuvry BJ, Tobias MA. Comparison of the antinociceptive activities of physostigmine, oxotremorine and morphine in the mouse. Br J Pharmacol, $43: 706-714,1971$.

34. Riding JE, Robinson JS. The safety of neostigmine. Anaesthesia, $16: 346-354,1961$.

35. Ris J, Lomholt B, Haxholdt O, Kehlet H, Valentin N, Danielsen V, Dyrberg V. Immediate and long-term mental recovery from general versus epidural anaesthesia in elderly patients. Acta Anaesthesiol Scand, 27 : 44-49, 1983.

36. Rinaldi $F$, Himwich HE. Alerting responses and actions of atropine and cholinergic drugs. AMA Arch Neurol Psychiat, 73 : 387-395, 1955.

37. Rosenzweig MR, Krech D, Bennett EL. A search for relations between brain chemistry and behaviour. Psychol Bull, 57 : 476-492, 1960.

38. Roy RC, Stullken EH. Electroencephalographic evidence of arousal in dogs from halothane after doxapram, physostigmine, or naloxone. Anesthesiology, 55 : 392-397, 1981.

39. RUPREHT $J$. Antagonism of ketamine by 4-AP and physostigmine. Br J Anaesth, 53 : 191-192, 1981.

40. Rupreht J, Jupa V. Physostigmine in the differential diagnosis of coma after neurosurgery. Acta Anaesthesiol Belg, 31 : 71-74, 1980.

41. Rupreht J, Dworacek B. Central anticholinergic syndrome in anesthetic practice. Acta Anaesthesiol Belg, 27: 45-60, 1976.

42. Salmenperä M, Nilsson E. Comparison of physostigmine and neostigmine for antagonism of neuromuscular block. Acta Anaesthesiol Scand, 25 : 387-390, 1981.

43. Snir-Mor I, Weinstock M, Davidson JT, Bahar M. Physostigmine antagonizes morphine-induced respiratory depression in human subjects. Anesthesiology, 59:6-9, 1983.
44. TAYlor P. Anticholinesterase agents (pp 100-119). In : Pharmacological basis of therapeutics, 6th edit, AG Gilman, LS Goodman and A Gilman eds. Macmillan, New York, 1980.

45. THESLEFF S. Aminopyridines and synaptic transmission. Neuroscience, $5: 1413-1419,1980$.

46. Thompson DEA. Physostigmine as an adjuvant to neuroleptanaesthesia in neurosurgical procedures. Can Anaesth Soc J, 23 : 582-586, 1976.

47. Varagic $V$. The action of eserine on the blood pressure of the rat. $\mathrm{Br} J$ Pharmacol, $19: 451-457,1955$.

48. Weinstock M, Erez E, Roll D. Antagonism of the cardiovascular and respiratory depressant effect of morphine in the conscious rabbit by physostigmine. $J$ Pharmacol Exp Ther, 218 : 504-508, 1981.

49. Weinstock M, Roll D, Erez E, Bahar M. Physostigmine antagonizes morphine-induced respiratory depression but not analgesia in dogs and rabbits. Br J Anaesth, $52: 1171$ 1175, 1980.

50. Weinstock M, Davidson JT, Rosin AJ, Schnieden H. Effect of physostigmine on morphine-induced postoperative pain and somnolence. Br J Anaesth, 54 : 429-432, 1982.

51. Wiener N, Deutsch JA. Temporal aspects of anticholinergic and anticholinesterase-induced amnesia for an appetitive habit. J Comp Psychol, 66 : 613-617, 1968.

52. WiKLER A. Pharmacologic dissociation of behavior and EEG sleep patterns in dogs : morphine, $N$-allylnormorphine and atropine. Proc Soc Exp Biol Med, 79 : 261-265, 1952 .

53. YAKSH TL. Direct evidence that spinal serotonin and noradrenaline terminals mediate the spinal antinociceptive effects of morphine in the periaqueductal gray. Brain Res, 160 : 180-185, 1979.

ABSTRACT: The central anticholinergic syndrome (CAS) includes central signs (somnolence, confusion, amnesia, agitation, hallucinations, dysarthria, ataxia, delirium, stupor, coma) and peripheral signs (dry mouth, dry skin, tachycardia, visual disturbances and difficulty in micturition). It occurs when central cholinergic sites are occupied by specific drugs and also as a result of an insufficient release of acetylcholine. The CAS can be caused by atropine sulphate, hyoscine (scopolamine), promethazine, benzodiazepines, opioids, halothane, influrane, ketamine. The incidence of CAS during the postoperative period depends on choice and dose of anaesthetic agents, type of surgery, patient's condition and diagnostic criteria. It is close to $10 \%$ following general anaesthesia and $4 \%$ following regional anaesthesia with sedation. The differential diagnosis of CAS includes an overdose of anaesthetic drugs or an alteration in pharmacokinetics, altered hydratation, electrolyte or acid-base state, hypoglycaemia, hypoxia, hypercapnia, hypocapnia, hyperthermia, hypothermia, hormonal disorders, neurological damage resulting from surgery, embolism, haemorrhage or trauma. The diagnosis of CAS is often determined by a process of exclusion and not actually made until a positive therapeutic response to physostigmine, a centrally active anticholinesterase agent has taken place. 